\title{
A PROCESS OF CREATING A SYSTEMATIC QoS ROUTING TECHNIQUE
} UTILIZING MANET

${ }^{1} \mathrm{R}$ Anitha, ${ }^{2} \mathrm{~B}$ R Tapas Bapu, ${ }^{3} \mathrm{~S}$ Soundararajan, ${ }^{4} \mathrm{M}$ Vengadapathiraj, ${ }^{5} \mathrm{~N}$ Partheeban, ${ }^{6} \mathrm{~V}$ Nagaraju

\author{
${ }^{1}$ Research Scholar of Anna University, Assistant Professor, S. A. Engineering College, Chennai. \\ ${ }^{2}$ Professor, Department of Electronics and Communication Engineering, S. A. Engineering College \\ Professor, Department of Computer Science and Engineering, Velammal Institute of Technology \\ Assistant Professor, Department of Electronics and Communication Engineering, Rajalakshmi Institute of Technology \\ ${ }^{5}$ Professor, School of Computing Science and Engineering, Galgotias University \\ Professor \& Head, Department of Electronics \& Communication Engineering, Rajalakshmi Institute of Technology
}

DOI: $10.51201 / J U S S T / 21 / 05194$

$$
\text { http://doi.org/10.51201/JUSST/21/05194 }
$$

\begin{abstract}
MANET is a mobile ad-hoc network that is made of several mobile nodes that can communicate in multi-way without any fixed or regular infrastructure. Due to its special features such as its self-organization, easy deployment it has been preferred for many military and civil applications. MANET has also gained popularity in the multimedia field. MANET has certain levels of requirements such as QoS (Quality of Service), jitter and energy, bandwidth, and end-2-end delay. MANET's one of the basic requirements is having QoS and should have efficient routing to support other applications. In this research paper, a special Genetic Algorithm known as the GA algorithm based on routing on a Mobile Ad-hoc network is designed and termed as GAMAN. The proposed model uses a 2 QoS system for routing. The outcome of this paper showed that the GAMAN method is a significant one for QoS in MANET.
\end{abstract}

Index Terms - Routing Model, QoS, MANET, GAMAN, mobile nodes

\section{INTRODUCTION}

Sensor nodes have a great capacity for which can identify and sent the corresponding data to the specific domain without any error. This is one of the innovation steps that has occurred in WSN. Sensor nodes are made up of certain features like sensing, which can process and transmit data which is the key component for running WSN. The nodes present in MANET can be used to communicate in a p-2-p fashion. Communication between 2 pairs of nodes is act as a router. In this case, the nodes will operate between the host and the routers. The network topology in MANET varies frequently and efficient work must be carried out in improving the MANET routing system. Many routing systems are not supporting QoS requirements. But it has gained more attention among the WSN domain. But the QoS cannot be directly applied to the MANET network due to constraints in bandwidth and varying network topology. The QoS based research on MANET supports have certain model they are

1. QoS model

2. QoS MAC

3. QoS reservation signaling

4. QoS Routing

MANET's topology should change as per the needs of the QoS if it is not changing then the process becomes meaningless. The proposed GAMAN should softly support the QoS without taking full control over the process in a hard guarantee manner. The QoS must look 
through the path being unbroken of any situation. It was found that many multimedia are largely accepting soft Quality of service and they use them in many ways. The proposed GAMAN algorithm utilizes 2 parameters they are the transmission and the delay parameter which helps in deciding the path of QoS.

\section{A. Related Work}

Figure 1 illustrates the MANET system. Figure 2 illustrates the QoS in MANET [11]. In this model, the mobile nodes are labeled as A, B, C, D, L etc. the number near the edge nodes shows the bandwidth of the wireless network. In case one needs to know the route from the SN the source node from mobile node A to the destination Node known as the DN suppose G. easy way to attain the destination will be preferred for instance the shortest route A-2-B-2-H-2-G will be selected to attain the DN. But this process is slightly different in the QoS method [7]. In QoS routing protocol the route from the source node A and the destination node $G$ the route selection will be based on minimum bandwidth [10]. The route here is from A,B,G. The shortest path will not be applicable because the capacity for providing the required bandwidth may not be possible all the time [5]. One of the significant goals of QoS is to find the determined path from the Source node to the destination node which should sacrifice its needs for attaining the desired QoS [13]. The route in QoS is based on certain conditions traffic and distance consideration, and minimal search.

\section{B. Proposed Algorithm}

In this model, it is assumed that there are possibilities for the host to communicate in the shared wireless channel. Each node has an identifier, 1 receiver, and 1 transmitter. Let's assume that the transmission distance for all the nodes is equal; When 2 nodes are neighbors, they will have a link between them only if they are in the same transmission range. The Neighbor discovery method may be applied in this model. Nodes will themselves transmit a BEACON to identify their own self. Through which they can easily identify their neighbor node. MAC protocol present in the system may help in resolving media contention, resource reservation, the neighbor node keeps the message and the other node placed near them will only discard the message. It is applicable only for a small network. For larger networks distributed or cluster- based algorithms may be used. let's us assume that the variation in topology may occur frequently. The topology change should occur after stability. This protocol gives more importance to the movable host than the steady host. 


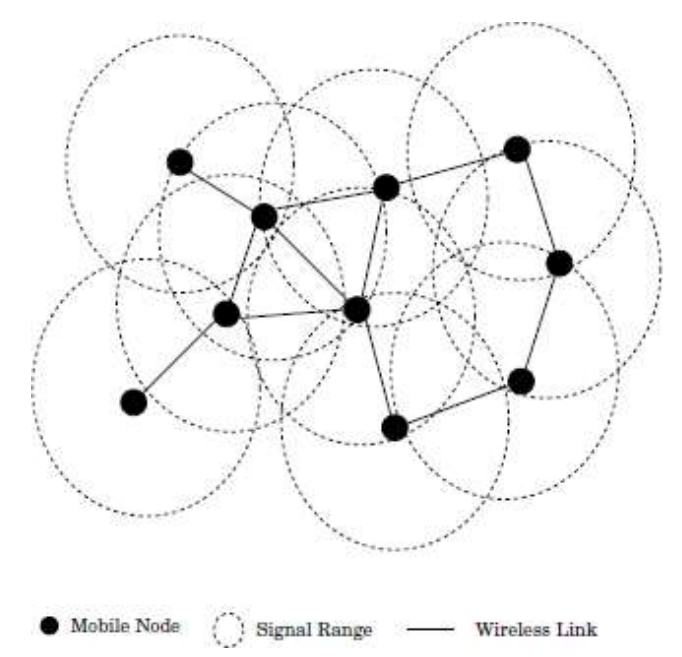

Fig. 1. A MANET

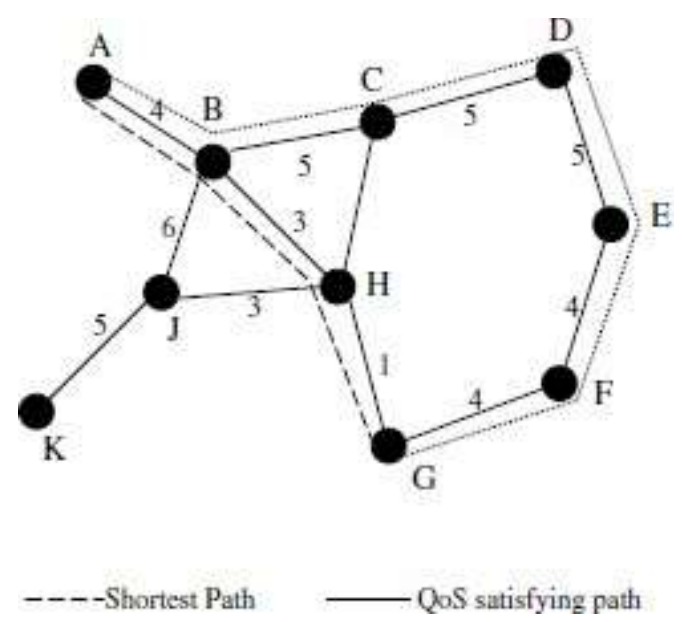

Fig. 2. An example of QoS in MANET

\section{GAMAN GOALS}

A. Certain goals have been set in this research for MANET routing protocol they are:

1) MANET's topology should change as per the needs of the QoS if it is not changing then the process becomes meaningless.

2) The proposed GAMAN should softly support the QoS without taking full control over the process in a hard guarantee manner.

3) The QoS must look through the path being unbroken of any situation.

4) It was found that many multimedia are largely accepting soft Quality of service and they use them in many ways.

5) The proposed GAMAN algorithm utilizes 2 parameters they are the transmission and the delay parameter which helps in deciding the path $f$ QoS.

B. The proposed GAMAN algorithm is made up of certain sets of features they are:

1) The proposed algorithm is a source-based routing algorithm

2) Certain nodes are used for routing computation based on the population size of the node

3) When considering the sub-population, the nodes placed in the sub-population will only care about the route in their sub-population 
4) The information broadcast is neglected here due to the restriction of passing information to the nodes

5) The GA search for all routes and the best route is selected based on a ranking system. The 1st rank route is the best one other route may be used as a backup one.

6) Loops in the system are rejected by using tree-shaped GA

7) In this proposed method the ETSA Effective topology search algorithm is used for extracting efficient topology in the MANET which helps in rejecting hidden terminal problems.

8) The motive of the proposed model is to give a good routing system and should be capable of acting to the queries of the system as early as possible.

\section{GAMAN ALGORITHM}

\section{A. GA cycle}

Fig 3 illustrates the GA cycle. At the initial stage of population proper solution was framed as a starting point for the search. The net stage was problem-solving which arise due to performance evaluation. The parent will be chosen based on the fitness of the individual they are selected over mutation and crossover operators. 2 chromosomes are selected by the crossover operator and genetic information of the chromosomes is swapped to produce a new chromosome.(Vimala et al., 2017)[13] In the mutation process the operator tries to bring in a new genetic structure this is done by modifying the gene also helps the search algorithm to escape from the pressure of local optimums. In this, the GA has the capability of changing the whole population or the limited member.

\section{B. Gene Coding}

Gene coding is one of the significant operations needed to perform in genetic operation which influences the significance of this operation.

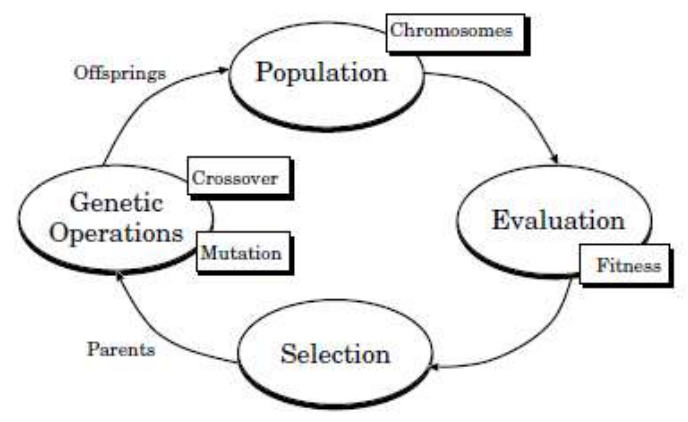

Figure 3. GA cycle 


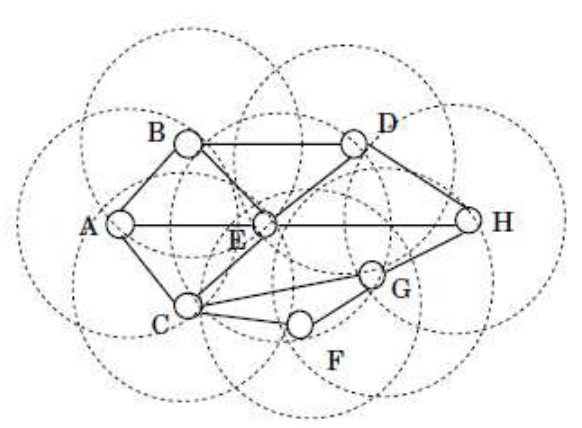

Figure 4. A MANET with 6 nodes

In the GAMAN algorithm, a tree-shaped model is used to simplify the network operation. The genes are placed at the tree junction. Where the length of the chromosome is similar and the genetic operatic takes place at the tree junction

This process is explained by considering a small MANET operation with 6 nodes as illustrated in fig 4. For instance, let's take node A which is the source node, and here $\mathrm{H}$ is the destination node. Fig 5 illustrates the route of the nodes in the network tree model. The shaded area is the one that describes the route from node $\mathrm{C}$ to node $\mathrm{H}$. the chromosome gene number of the node is reduced as illustrated in fig 6 . In the reduced network model where all the tree junction is taken as the gene and the path for the destination is shown by the chromosome.

Fig 7 illustrates the chromosome route of nodes from $A$ to $H$. in the proposed GAMAN model the chromosome has 2 states known as the active state and the inactive state.

\section{GAMAN Operation}

The 2 parameters of QoS is the DT known as the delay time and the TSR known as the Transmission success rate is used by GAMAN. DT is the time taken for a packet to reach from 1 node to another. TSR is the rate of transmitted packets correctly. The T value is shown as

$$
=\frac{\sum_{i=1}^{n} D T_{i}}{\prod_{i=1}^{n} T S R_{i}}
$$

Where $\mathrm{n}$ is the number of wireless links in a path.

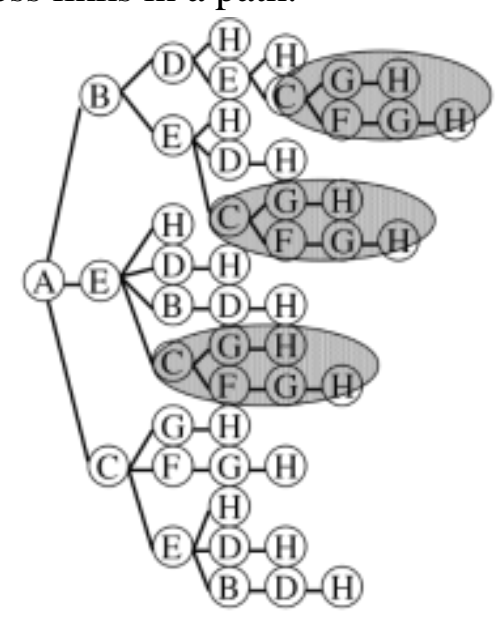

Figure 5. Network tree model 


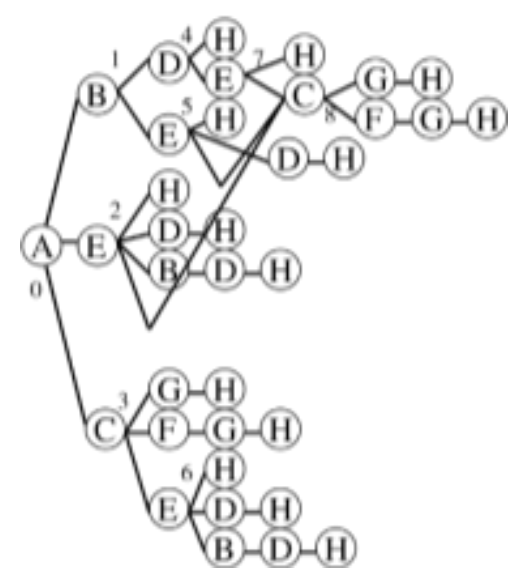

Figure 6. Reduced network tree model

After the reduction of the network model, the routes for reaching the path is missing. The reduction model helps in reducing the length of the chromosome and the genetic operation becomes simpler. The tree junction in the reduced model is coded as the gene. The gene knows the information about the adjacent nodes of a chromosome. Once the gene coding is complete the GAMAN will ensure to start the genetic operation (GO). In this operation in the initial stage, the initial population for the study will be selected. Form the selected population 2 individual will be chosen to perform a ranking function to carry out GO. The ranking here is based on the fitness. Fitness completely relies on the value of T. it is found which less $\mathrm{T}$ value the fitness of the individual will be more. The GO is the mutation operators (MO) and the crossover operators (CO). In GAMAN 1-point CO is used for the quick response. In $\mathrm{MO}$ the gene are selected on a random basis based on probability mutation $\leq 1 / l$, where $l$ is the length of the chromosome. After the completion of $\mathrm{CO}$ and $\mathrm{MO}$, the Elitist model (EM) is utilized from the EM the individual with high fitness is identified.

\begin{tabular}{|c|c|c|c|c|c|c|c|c|}
\hline 0 & 1 & 2 & 3 & 4 & 5 & 6 & 7 & 8 \\
\hline BEC & $\mathrm{DE}$ & $\begin{array}{l}\text { HD } \\
\text { BC }\end{array}$ & GFE & $\mathrm{HE}$ & HDC & HDB & $\mathrm{HC}$ & GF \\
\hline
\end{tabular}

Figure 7. GAMAN gene coding

Table 1. The number of nodes, routes, and branches

\begin{tabular}{|c|c|c|c|c|}
\hline Nodes & 10 & 20 & 30 & 35 \\
\hline Routes & 36 & 725 & 11375 & 23076 \\
\hline Branches & 13 & 33 & 85 & 246 \\
\hline
\end{tabular}

The GO will be repeated until getting a route with less $\mathrm{T}$ value. In GAMAN route selection is completely based on value of $\mathrm{T}$. T is the ratio of DT along with TSR. It is found that by minimizing the value of T the value of DT will be minimized through which the value of TSR will be maximized. Through which it is understood that the packet where transmitted from the source node to destination node will small delay but by giving a high TSR. GAMAN will be used for small MANET. But for large MANET distributed or cluster-based 
method will be preferred for routing.

\section{SIMULATION RESULTS}

In this many simulations have been carried out for various topologies with several routes, branches, and nodes. This process is shown in table 1 as follows. For this process, a MANET with 18 nodes is selected. In between the process, the nodes number was decreased to 10 then in a later stage the number of nodes was between 30 and 40 .

In the initial stage, a random path was chosen for TSR and DT for each and every wireless MANET link. Then the $\mathrm{T}$ value was calculated along with the ratio of DT and TSR. The speeds have taken by GAMAN to find a new route was investigated. The GO was repeated until getting the small value of $\mathrm{T}$.

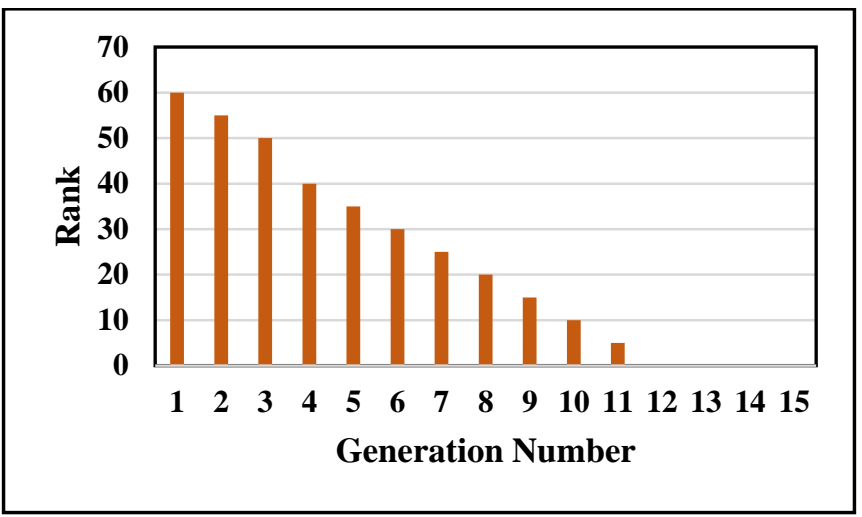

Figure 8. Performance behavior of GAMAN algorithm

Table 2. The time needed for one generation (ms).

\begin{tabular}{|c|c|c|c|}
\hline Nodes & Average & Max & Min \\
\hline 10 & 4.20 & 16 & $*$ \\
\hline 20 & 4.80 & 20 & $*$ \\
\hline 30 & 6.41 & 30 & $*$ \\
\hline 35 & 10.71 & 50 & $*$ \\
\hline
\end{tabular}

Table 3. Performance for different parameters.

\begin{tabular}{|c|c|c|c|}
\hline Nodes & Rank & Gen & Ref \\
\hline 10 & 2.64 & 1.00 & 10.00 \\
\hline 20 & 5.62 & 8.00 & 26.30 \\
\hline 30 & 6.44 & 42.30 & 80.84 \\
\hline 35 & 5.38 & 28.72 & 65.62 \\
\hline
\end{tabular}

Table 4. Comparison between GAMAN and GAMAN1

\begin{tabular}{|c|c|c|c|c|}
\hline Method & Ta & DA & TSRA & GSA \\
\hline GAMAN & 4.47 & 10.52 & 9.36 & 9.00 \\
\hline GAMAN1 & - & 4.66 & 70.66 & 8.33 \\
\hline
\end{tabular}

Where DAthe average rank value of delay, TSRA is is the average rank value of the TSR parameter, GSA is the average value of generation number, GOTA is the average value 
of genetic processing time.

From the comparative study, it was found that the GO was better in GAMAN-1 when compared to GAMAN. From this study, it was also found that when we use 1 QOS parameter the performance was a little dull. So it is preferred to use both the QoS parameter instead of using one of them.

\section{CONCLUSIONS}

In this research paper, a new type of quality-of-service approach was used for MANET. From the simulation result, it was found that the GAMAN is one the significant one for quality-of-service provider for the MANET network. GAMAN is having a proper response for the time. It has significant GENE coding skills and is capable of supporting the QoS parameters. From the comparative study, it was found that GAMAN can find the quality-of-service route than the GAMAN-1. The proposed GAMAN can be used for the network which is smaller in scale. For a network with a large scale, a new method must be proposed which is an alternative to the GA model.

\section{REFERENCES}

[1] S. Vimala, V. Dr, H. Khanna, and Venkateswaran, "Multicast Optimal Energy Aware Routing Protocol for Manet Based on Swarm Intelligent Techniques," International Journal of Civil Engineering and Technology, vol. 8, pp. 1-1, 2017.

[2] A. Sodhro and Hassan, "Quality of service optimization in an iot- driven intelligent transportation system," IEEE Wireless Communica-tions, vol. 26, pp. 10-17, 2019.

[3] S. Ahmed and Imroze, TRAFFIC AWARE ROUTING ALGORITHMS FOR ENERGY EFFICIENT OPTICAL-WIRELESS ACCESS NET-WORKS. Diss, 2019.

[4] M. Khan, I. Firdaus, and Das, "A study on quality-of-service routing protocols in mobile ad hoc networks," 2017 International Conference on Computing and Communication Technologies for Smart Nation (IC3TSN), 2017.

[5] M. Hassan and Hamid, "Integrating African Buffalo optimization algo- rithm in AODV routing protocol for improving the QoS of MANET," Journal of Southwest Jiaotong University, vol. 54, no. 3, 2019.

[6] W. Alnumay, U. Ghosh, and P. Chatterjee, "A Trust-Based Predictive Model for Mobile Ad Hoc Network in Internet of Things," Sensors, vol. 19, no. 6, pp. 
1467-1467,2019.[Online].Available: 10.3390/s19061467; https://dx.doi.org / 10.3390 / s19061467

[7] R. Arnous, E.-S. M., and M. Saber, "A Proposed Routing Protocol for Mobile Ad Hoc Networks," International Journal of Computer Applications, vol. 178, no. 41, pp. 26-30, 2019. [Online]. Available: 10.5120/ijca2019919305 https://dx.doi.org / 10.5120 / ijca2019919305.

[8] G. Gebreslassie, L. M. Immaculate, S. R. Sheela, and Grace, "Analyzing and Optimizing of Quality of Service Management in Flying Ad hoc Networks," International Science and Technology Journal, 2018.

[9] U. Baroudi, "Ticket-based QoS routing optimization using genetic algorithm for WSN applications in smart grid," Journal of Ambient Intelligence and Humanized Computing, vol. 10, pp. 1325-1338, 2019.J. W. Guck, A. V. Bemten, M. Reisslein, and W. Kellerer, "Unicast QoS Routing Algorithms for SDN: A Comprehensive Survey and Performance Evaluation," IEEE Communications Surveys \& Tutorials, vol. 20, no. 1, pp. 388-415, 2018. [Online]. Available:10.1109/comst2017.2749760; https://dx.doi.org / 10.1109 / comst.2017.274976

[10] B. R. T. Bapu and L. C. S. Gowd, "Link Quality Based Opportunistic Routing Algorithm for QOS: Aware Wireless Sensor Networks Security," Wireless Personal Communications, vol. 97, no. 1, pp. 1563- 1578, 2017. [Online]. Available: 10.1007/s11277-017-4586-4; https://dx.doi.org/10.1007/s11277-017-4586-4

[11] M. Usman, "QASEC: A secured data communication scheme for mobile Ad-hoc networks," Future Generation Computer Systems, vol. 109, pp. 604-610, 2020.

[12] N. Sivapriya and T. N. Ravi, "QoS Routing Protocols in MANET: A Survey," International Journal of Pure and Applied Mathematics, vol. 119, pp. 16 573-16 579, 2018.

[13] B. R. TapasBapu, V. Nagaraju, M. Ramesh, S. K. R. Kanna, and P. Sailaja, "Energy Efficient Wireless Sensor Network to Enhance Network Security by Detecting Clone Nodes," IOP Conference Series: Materials Science and Engineering, vol. 993, pp. 012 067-012 067, 2020. [Online]. Available: 10.1088/1757-899x/993/1/012067;https://dx. 